\title{
BMJ Open Rapid diagnostic test for antenatal syphilis screening in low-income and middle-income countries: a systematic review and meta-analysis
}

\author{
Carmen Phang Romero Casas, , ${ }^{1,2}$ Marrissa Martyn-St James, ${ }^{3}$ Jean Hamilton, ${ }^{3}$ \\ Daniel S Marinho, ${ }^{1,2}$ Rodolfo Castro, ${ }^{2,4,5}$ Sue Harnan ${ }^{3}$
}

To cite: Phang Romero Casas C, Martyn-St James M, Hamilton J, et al. Rapid diagnostic test for antenatal syphilis screening in low-income and middle-income countries: a systematic review and meta-analysis. BMJ Open 2018;8:e018132. doi:10.1136/ bmjopen-2017-018132

- Prepublication history and additional material for this paper are available online. To view these files, please visit the journal online (http://dx.doi. org/10.1136/bmjopen-2017018132).

Received 9 June 2017 Revised 31 0ctober 2017 Accepted 20 November 2017

Check for updates

${ }^{1}$ Centro de Desenvolvimento Tecnológico em Saúde (CDTS), Fundação Oswaldo Cruz (FIOCRUZ), Rio de Janeiro, Rio de Janeiro, Brazil

${ }^{2}$ National Institute for Science and Technology on Innovation on Diseases of Neglected Populations (INCT/IDPN), National Council for Scientific and Technological Development (CNPq), Brazil

${ }^{3}$ School of Health and Related Research, University of Sheffield, Sheffield, UK

${ }^{4}$ Instituto Nacional de Infectologia Evandro Chagas (INI), Fundação Oswaldo Cruz (FIOCRUZ), Rio de Janeiro, Brazil ${ }^{5}$ Instituto de Saúde Coletiva, Universidade Federal do Estado do Rio de Janeiro (UNIRIO), Rio de Janeiro, Brazil

Correspondence to Dr Carmen Phang Romero Casas; carmenprc@gmail.com

\section{ABSTRACT}

Objectives To undertake a systematic review and meta-analysis to evaluate the test performance including sensitivity and specificity of rapid immunochromatographic syphilis (ICS) point-of-care (POC) tests at antenatal clinics compared with reference standard tests (non-treponemal (TP) and TP tests) for active syphilis in pregnant women. Methods Five electronic databases were searched (PubMed, EMBASE, CRD, Cochrane Library and LILACS) to March 2016 for diagnostic accuracy studies of ICS test and standard reference tests for syphilis in pregnant women. Methodological quality was assessed using QUADAS-2 (Quality Assessment of Diagnostic Accuracy Studies). A bivariate meta-analysis was undertaken to generate pooled estimates of diagnostic parameters. Results were presented using a coupled forest plot of sensitivity and specificity and a scatter plot.

Results The methodological quality of the five included studies with regards to risk of bias and applicability concern judgements was either low or unclear. One study was judged as high risk of bias for patient selection due to exclusion of pregnant women with a previous history of syphilis, and one study was judged at high risk of bias for study flow and timing as not all patients were included in the analysis. Five studies contributed to the meta-analysis, providing a pooled sensitivity and specificity for ICS of 0.85 (95\% Crl: 0.73 to 0.92$)$ and 0.98 (95\% Crl: 0.95 to 0.99 ), respectively.

Conclusions This review and meta-analysis observed that rapid ICS POC tests have a high sensitivity and specificity when performed in pregnant women at antenatal clinics. However, the methodological quality of the existing evidence base should be taken into consideration when interpreting these results.

PROSPERO registration number CRD42016036335.

\section{INTRODUCTION}

Syphilis is a chronic infectious disease, caused by the spirochete bacterium Treponema pallidum, transmitted sexually (acquired syphilis) and vertically (congenital syphilis) through the mother's placenta to the fetus. ${ }^{1-3}$ Syphilis is among the high-risk conditions during pregnancy with possible serious birth outcomes if untreated or inadequately treated. ${ }^{14}$
Strengths and limitations of this study

- This systematic review addresses the performance of rapid tests to tackle significant public health problems that are maternal and congenital syphilis.

- The systematic review was conducted and presented in accordance with recognised standards for undertaking and reporting systematic reviews and

- Methodological quality of studies was assessed using a validated method.

- Data were synthesised using a bivariate randomeffects meta-analysis model, implemented in a Bayesian framework.

- There were insufficient data from the included studies to undertake subgroup analyses.

WHO estimates that syphilis infects $1 \%$ or more of antenatal care attendees in over 55 countries. Approximately 950000 pregnant women were infected with syphilis in $2012,{ }^{5}$ primarily in the low-income and middle-income countries, sub-Saharan Africa, Southeast Asia and Latin America and the Caribbean (LAC). The prevalence of gestational syphilis in LAC varies from $0.08 \%$ to $7.0 \%{ }^{67}$ between countries, and accounts for up to $25 \%$ of the 2 million annual cases of gestational syphilis in these regions. ${ }^{6} 7$ Annually, an estimated 100000 stillbirths in these regions are attributable to congenital syphilis. ${ }^{67}$

Diagnosis of syphilis is complicated due to extremely difficult to cultivate $T$. pallidum in vitro. Clinical diagnosis, visualisation of treponemes in lesion material using darkfield microscopy or immunofluorescent staining and serological reactions are used for diagnosis. Nevertheless, serological tests are a mainstay of syphilis diagnosis. ${ }^{89}$

Syphilis can be diagnosed serologically by using a non-treponemal (non-TP) test for screening and a TP test for the confirmation of results. TP tests are more sensitive and 
more specific than non-TP tests, but they cannot distinguish between active and past infection, as antibodies remain circulating during life after treatment. Non-TP tests provide high sensitivity but not for the initial stage of primary syphilis or late period of disease (tertiary syphilis) ${ }^{8-10}$

In low-income and middle-income countries, many prenatal clinics that provide screening and treatment for syphilis do not have the capability to perform confirmatory diagnostic tests, and so testing is frequently done off-site. ${ }^{11}$ Furthermore, patients may fail to return for laboratory results and both specimens and results have the potential to be lost in transit, resulting in treatment being delayed or missed.

Currently, new tests using cloned TP antigens and an immunochromatographic technique provide an alternative platform of rapid TP testing to be performed at point-of-care (POC), this is the immunochromatographic syphilis (ICS) test. The ICS test takes approximately $20 \mathrm{~min}$ to perform and is generally considered fairly sensitive and specific. ${ }^{8}$ In addition, the format of the test does not require the use of special equipment such as fluorescence microscopes or microplate readers, thus making it an ideal test for POC settings. ${ }^{12}$

A rapid syphilis test with immediate results implemented in a field setting has the advantage of allowing women who test positive to be treated on-site at the same visit, avoiding losses to follow-up for return visits and potential adverse outcomes associated with delayed treatment. ${ }^{13}$ According to the Special Programme for Research and Training in Tropical Diseases (WHO) ${ }^{14}$ the ideal characteristics of POC tests are that they are affordable, sensitive, specific, user- friendly, rapid and robust. ${ }^{15}$ On-site screening services can contribute to significant declines in the prevalence of maternal and congenital syphilis in many low-income and middle-income countries, ${ }^{16}$ where operational research on POC syphilis testing is already underway. ${ }^{17}$

This systematic review and meta-analysis of diagnostic test accuracy aims to evaluate the performance of rapid ICS POC test at antenatal clinics compared with reference standard tests (non-TP and TP tests) for detecting active syphilis in pregnant women. Furthermore, in comparison to previous reviews by Tucker et $a l^{17}$ and Jafari $e t \mathrm{al}^{18}$ that included studies among women attending sexually transmitted infection (STI) clinics and antenatal clinics, the present systematic review includes only studies among pregnant women in antenatal care settings, that compare ICS rapid test with the algorithm established as reference standard (non-TP, following by TP test) ${ }^{10}$ used in many low-income and middle-income countries.

\section{METHODS}

\section{Protocol and guidelines}

The protocol for this review can be found at PROSPERO 2016: CRD42016036335, available from http://www.
crd.york.ac.uk/PROSPERO/display_record.asp? ID= CRD42016036335.

The systematic review was conducted and presented in accordance with the general principles recommended in the Preferred Reporting Items for Systematic Reviews and Meta-Analyses (PRISMA) statement. ${ }^{19}$

\section{Search strategy}

PubMed, EMBASE, CRD, Cochrane Library and LILACS (Literatura Latinoamericana en Ciencias de la Salud) were searched initially from January 1960 to end March 2016 and were updated to end August 2017. The following keywords were used: 'pregnancy diseases; maternal syphilis; gestational syphilis; congenital syphilis' and 'diagnostic; screening; rapid test; serological test(s); point-of-care' and 'accuracy; sensitivity, specificity'. The update search also included the term 'antenatal'. Full search strategies for PubMed and EMBASE databases are presented in online supplementary appendix 1 . Filters for diagnostic studies recommended by Information Specialists' Sub-Group Search Filter Resource (https://sites. google.com/a/york.ac.uk/issg-search-filters-resource/) were used. No language restrictions were applied to the search. Reference lists of all included studies were also searched.

\section{Inclusion criteria for studies}

Diagnostic test accuracy studies were included if they evaluated ICS POC tests for syphilis, compared with a standard reference test in pregnant women who receive antenatal care. We only included studies where the standard reference test comprised a non-TP test (Venereal Disease Research Laboratory (VDRL) or rapid plasma reagin (RPR)) followed by a TP test (T. pallidum haemagglutination test (TPHA), or fluorescent treponemal antibody absorbed (FTA-Abs), or T. pallidum particle agglutination (TPPA)). The standard reference test constitutes a non-TP test, followed by TP test where the non-TP test is positive. We also only included studies reporting test performance data (true positive, false positive, false negative, true negative) from which to calculate sensitivity and specificity, or other information from which we were able to calculate performance data. Dual tests such as HIV/ syphilis tests and TP/non-TP POC tests were not included in this review.

\section{Study selection process}

All citations were downloaded to Mendeley reference management software (https://www.mendeley.com/ newsfeed/). Titles and abstracts were sifted by one reviewer (CNPRC) to identify potentially relevant articles. All potentially relevant articles obtained as full text were checked for inclusion by two reviewers (CNPRC, SH).

\section{Data extraction}

Data were extracted by CNPRC using a prepiloted data extraction form in Excel and then checked by two independent reviewers (RC and DSM). Any discrepancies were resolved by a third reviewer (MMS-J or SH). Details 
of study characteristics, population characteristics, test descriptions and performance data outcomes (true positives, false positives, false negatives and true negatives; plus sensitivity and specificity) were extracted from the included studies.

\section{Assessment of methodological quality}

The quality of evidence was assessed and described using the Bristol University QUADAS-2 (Quality Assessment of Diagnostic Accuracy Studies) tool to analyse risk of bias. ${ }^{20}$

QUADAS-2 is structured in four domains of potential sources of bias in primary diagnostic studies: 'patient selection; index test; reference standard and flow and timing'. There are signalling questions within each of these domains, which drive researchers to overview the potential sources of bias. ${ }^{20}$

\section{Data analysis}

The extracted data and quality assessment variables were presented for each study, both in structured tables and as a narrative description. The combined sensitivity and specificity was estimated using the bivariate random-effects meta-analysis model described by Reitsma et al. ${ }^{21}$ The data synthesis was conducted under the assumption that different specific implementations of ICS rapid tests (eg, by different manufacturers) can be considered equivalent. Therefore, there is essentially one index test only. A random-effects model was used to allow for heterogeneity between studies that is generally expected in studies of diagnostic test accuracy, ${ }^{22}$ which in this case will include heterogeneity due to differences in the specific index test used. Further details of the statistical model are provided in online supplementary appendix 2. Results were displayed as forest plots and scatter plots with $95 \%$ credible intervals (CrIs) and 95\% prediction intervals (PrIs) for sensitivity and specificity.

Analyses were conducted in the $\mathrm{R}$ software environment $^{23}$ using WinBUGS ${ }^{24}$ and R2WinBUGS software packages. ${ }^{25}$ Convergence to the target posterior distributions was assessed using the Gelman-Rubin convergence statistic. ${ }^{26}$ A burn-in of 10000 iterations of the Markov chain was used with a further 10000 iterations retained to estimate parameters.

\section{RESULTS}

\section{Study selection process}

The results of the study selection process are illustrated in figure 1 using a PRISMA flow diagram. ${ }^{19}$ Following deduplication, the searches identified 484 citations of which 28 were obtained for screening. Of these, 23 full-text publications were excluded. A table of these excluded studies with reasons for exclusion is presented in online supplementary appendix 3. Five diagnostic accuracy studies were included in the review.

\section{Characteristics of the included studies}

The characteristics of the five included studies, including the study setting, study design, details of included participants and details of the ICS and non-TP/TP tests are presented in online supplementary appendix 4 .

All of the included studies were prospective, singlegate (a study design in which only suspected patients for the target condition are recruited) diagnostic accuracy studies. Four studies reported consecutive sampling. ${ }^{27-30}$ The sampling method was not reported in one study. ${ }^{31}$ All included studies were undertaken in antenatal clinics ${ }^{27-29}$ or hospitals. ${ }^{30} 31$

All the studies were undertaken in low-income and middle-income countries (Brazil, South Africa, Mexico, India, Mozambique and Bolivia) according to the World Bank country classification. ${ }^{32}$ Two were undertaken in urban health centres ${ }^{3031}$ and three at rural sites. ${ }^{27-29}$

Across the included studies the index tests used were ICS tests. Two studies ${ }^{28}{ }^{30}$ used the Determine Syphilis TP test (Abbott Laboratories, Abbott Park, Illinois, USA), two studies $^{29}{ }^{31}$ used the SD Bioline V.3.0 test (Standard Diagnostics, Kyonggi-Korea) and one study ${ }^{27}$ used the VisiTect Syphilis test (Omega Diagnostics, Alloa, Scotland).

These ICS tests detect IgM, IgG and IgA antibodies and involve ICS in which one or multiple T. pallidum recombinant antigens are applied to nitrocellulose strips as a capture reagent. Antibodies in the specimen bound at antigen site on the strip and are revealed with dye bound anti-immunoglobulin or dye bound antigen and a positive reaction appears as a coloured line. ${ }^{9}$ The individual tests are also very similar in the assay procedure, taking from 20 to $30 \mathrm{~min}$ to get the result.

The age of participants was reported by three included studies, ${ }^{27} 2931$ in which all women were in the third decade.

Across the five studies in pregnant women, three studies reported that participants were in the second trimester $^{27-29}$ and one study reported that $16 \%, 32 \%$ and $52 \%$ of participants were in the first, second and third trimester, respectively. ${ }^{31}$ One study in pregnant women did not report the stage of pregnancy. ${ }^{30}$

Obstetric characteristics were reported by two studies ${ }^{2729}$ identifying: previous syphilis $(\mathrm{n}=4 ; 2.1 \%)^{27}$ and $\mathrm{n}=318 ; 7.1 \%)$, ${ }^{29}$ previous treatment for syphilis, including penicillin $(\mathrm{n}=51 ; 7.5 \%)$, a previous syphilis diagnosis $(\mathrm{n}=318 ; 7.1 \%)^{27}$ and prior partner syphilis history (as reported by the pregnant women $)(\mathrm{n}=228 ; 5.3 \%) .{ }^{29}$ Three studies did not report obstetric characteristics. ${ }^{28} 3031$

For the Bronzan et $a l^{28}$ study, we analysed on-site ICS results for all the study participants $(n=695)$ instead of on-site ICS results after training on how to properly obtain fingerstick blood $(n=341)$. This study reported sensitivity and specificity both before and after nursing training on how to properly obtain fingerstick blood, $\left(31.3 \%-85.7 \%\right.$ and $94.8 \%-90.9 \%$, respectively) ${ }^{28}$ None of the other included studies reported whether nurse training on how to properly obtain fingerstick blood was provided or not. As the inclusion criteria for studies, specified in the review protocol for this review, were not 


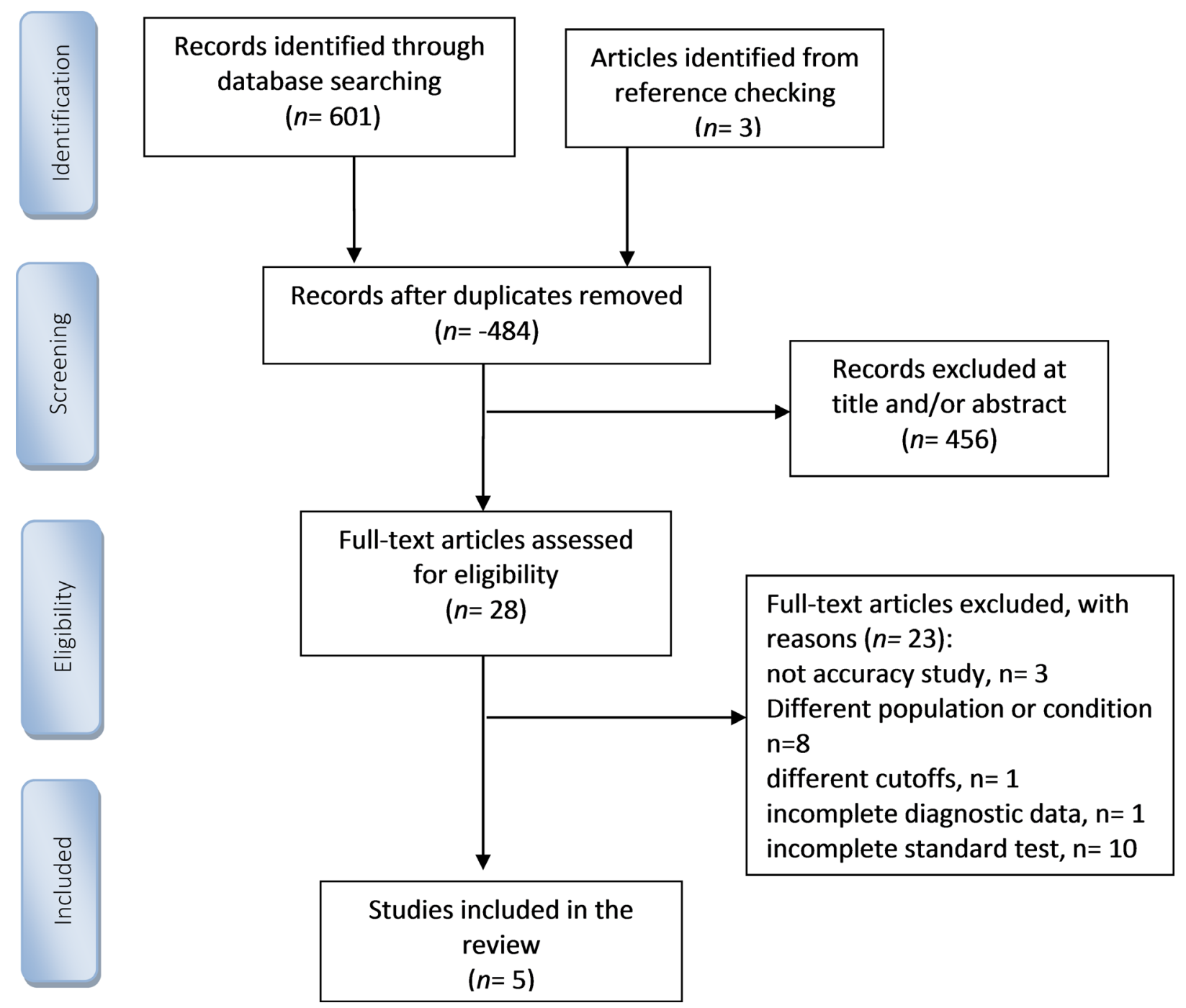

Figure 1 Study selection following Preferred Reporting Items for Systematic Reviews and Meta-Analyses guidelines. ${ }^{19}$

based on whether training was provided or not, it was considered most appropriate to include results over the whole study period. Meta-analysis results including data from the Bronzan $e t a l^{28}$ study after retraining on how to properly obtain fingerstick blood are also displayed for comparison.

In the Kashyap et $a \ell^{11}$ study, all patients received both VDRL and TPHA tests, however, TPHA was the confirmatory test, and it was not clear how VDRL-negative/ TPHA-positive tests were interpreted. The authors were contacted for clarification, but without response to date.

\section{Methodological quality of included studies}

The overall methodological quality (risk of bias and applicability concerns) of the five included studies assessed by QUADAS-2 is summarised in figure 2. Kashyap $e t a l^{31}$ was judged as high risk of bias and high concern for patient selection as the study excluded pregnant women with previous history of syphilis. Montoya $e t a l^{29}$ was judged at high risk of bias for study flow and timing as not all patients were included in the analysis. The remaining risk of bias and applicability concern judgements across studies were either low or unclear.

\section{Results reported by included studies}

The diagnostic accuracy data reported by the five included studies are presented in table 1 .

\section{Results of the meta-analysis}

Five studies (14985 participants) contributed to the meta-analysis. Figure 3 shows the sensitivity and specificity for each study, as estimated by the model. Note that the modelled estimates of sensitivity and specificity for each study are shrunken towards the overall mean, and hence differ from the raw estimates presented in table 1 . The pooled sensitivity and specificity of ICS POC test compared with a standard reference test were 0.85 (95\% CrI 0.73 to 0.92 ) and 0.98 (95\% CrI: 0.95 to $0.99)$. The between-study SD for sensitivity and specificity were estimated to be 0.58 (95\% CrI: 0.33 to 1.15 ) and 0.71 (95\% CrI: 0.42 to 1.47 ), respectively. The correlation between logit sensitivity and specificity was not statistically significant 0.42 (95\% CrI: -0.46 to 0.88 ). The $95 \%$ PrIs 


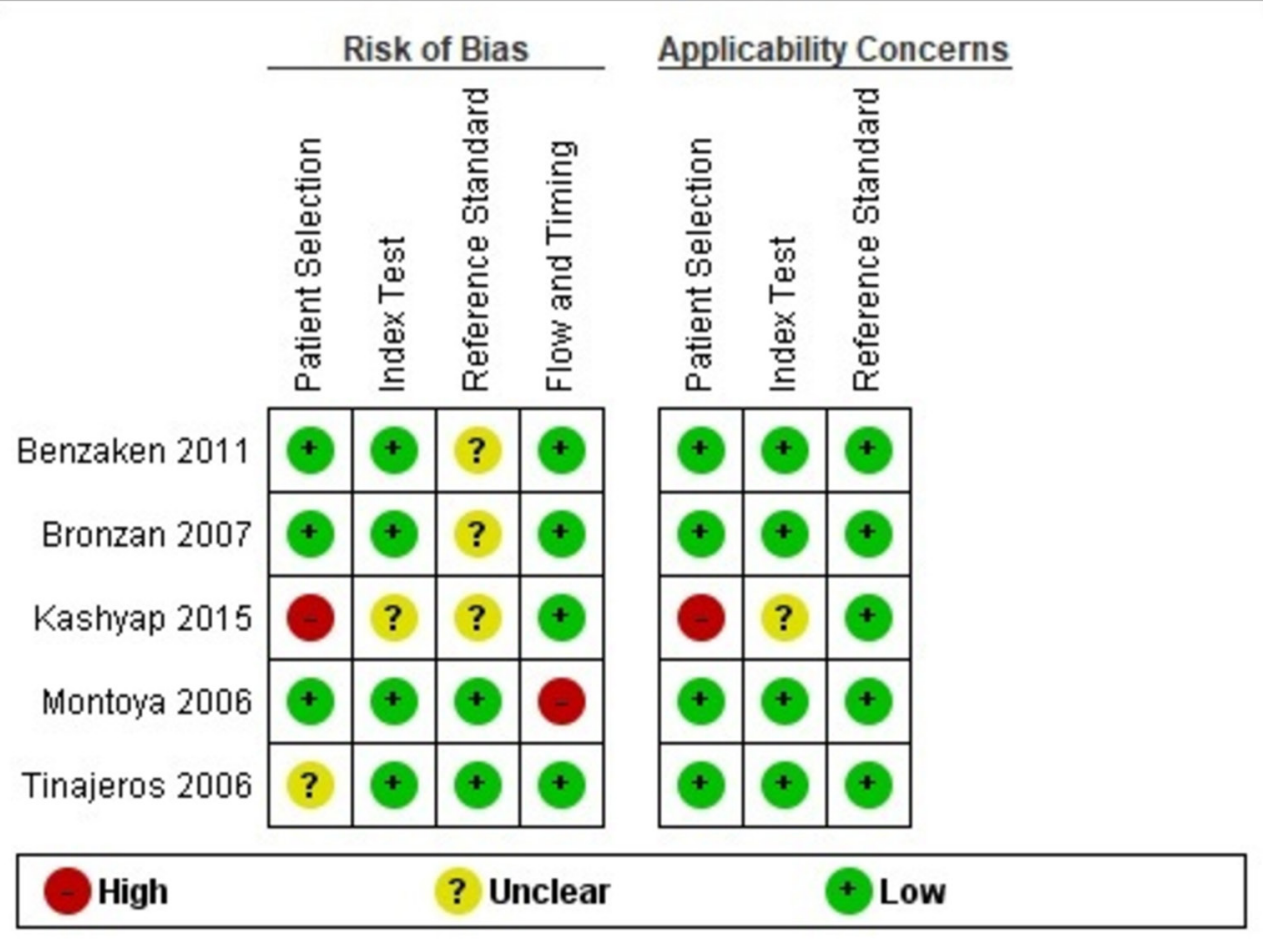

Figure 2 Risk of bias and applicability concerns summary.

of 0.57 to 0.96 (sensitivity) and 0.88 to 1.00 (specificity) suggest moderate uncertainty in predicting the sensitivity and specificity of a new study. Figure 4 presents the joint distribution for sensitivity and specificity.

Two sensitivity analyses were conducted. Assuming that missing test results in Benzaken $e t a l^{27}$ were negative (rather than positive) reduced the pooled estimate of the sensitivity only slightly to 0.84 (95\% CrI: 0.71 to 0.92 ) (online supplementary figure 1 ). The impact of retraining was assessed by including the data after training only from Bronzan $e t a l,{ }^{28}$ resulting in slightly higher sensitivity of 0.88 (95\% CrI: 0.78 to 0.93 ) (online supplementary figure 2). See online supplementary appendix 5.

\section{DISCUSSION}

This systematic review of the evidence for the diagnostic accuracy of TP antibody-based ICS tests among pregnant women with active syphilis included five studies (14985 participants). Although the ICS tests employed by the included studies are produced by different manufacturers, they are all similar in their implementation. As such, in order to assess the accuracy of ICS tests, estimates from all tests were pooled together in the meta-analysis.

We used an established algorithm of POC without confirmatory non-TP as a reference standard where a positive result indicates that immediate treatment is required for pregnant women. ${ }^{9}$ The meta-analysis was conducted under the assumption that the reference standard is $100 \%$ sensitive and specific.

The meta-analysis suggested a high average sensitivity 0.85 (95\% CrI: 0.73 to 0.92 ) and specificity 0.98 (95\%
CrI: 0.95 to 0.99 ) of the ICS tests, compared with standard tests (non-TP test followed by TP test where the non-TP test is positive). The high sensitivity of the ICS test indicates that it might be a reasonable initial screening test for syphilis, but subsequent clinical management would require a non-TP test. Overall, we observed that rapid and POC tests performed well in both sensitivity and specificity compared with laboratory-based TP-specific tests such as TPPA and TPHA that have sensitivity in the range of $85 \%-100 \%$ and specificity of $98 \%-100 \% .^{33}$

The risk of bias and concerns over applicability of the five studies was either low or unclear. One study was judged as high risk of bias and high concern for patient selection as the study excluded pregnant women with previous history of syphilis. ${ }^{31}$ Another study was judged at high risk of bias for study flow and timing as not all patients were included in the analysis. ${ }^{29}$ The contribution of these studies to the pooled analysis should therefore be taken into consideration. A major limiting factor in assessing methodological quality was unclear or incomplete reporting by authors.

We undertook our review according to PRISMA guidelines, searched a wide range of data sources, undertook double data checking and assessed the methodological quality of the included studies using QUADAS-2. However, the presented review also has limitations. Although HIV status, clinical stage of syphilis infection and syphilis titre might influence ICS test performance, ${ }^{8}$ there were too few included studies, and insufficient data available from the included studies for us to explore these potential sources of heterogeneity. In one of the included studies, ${ }^{31}$ 


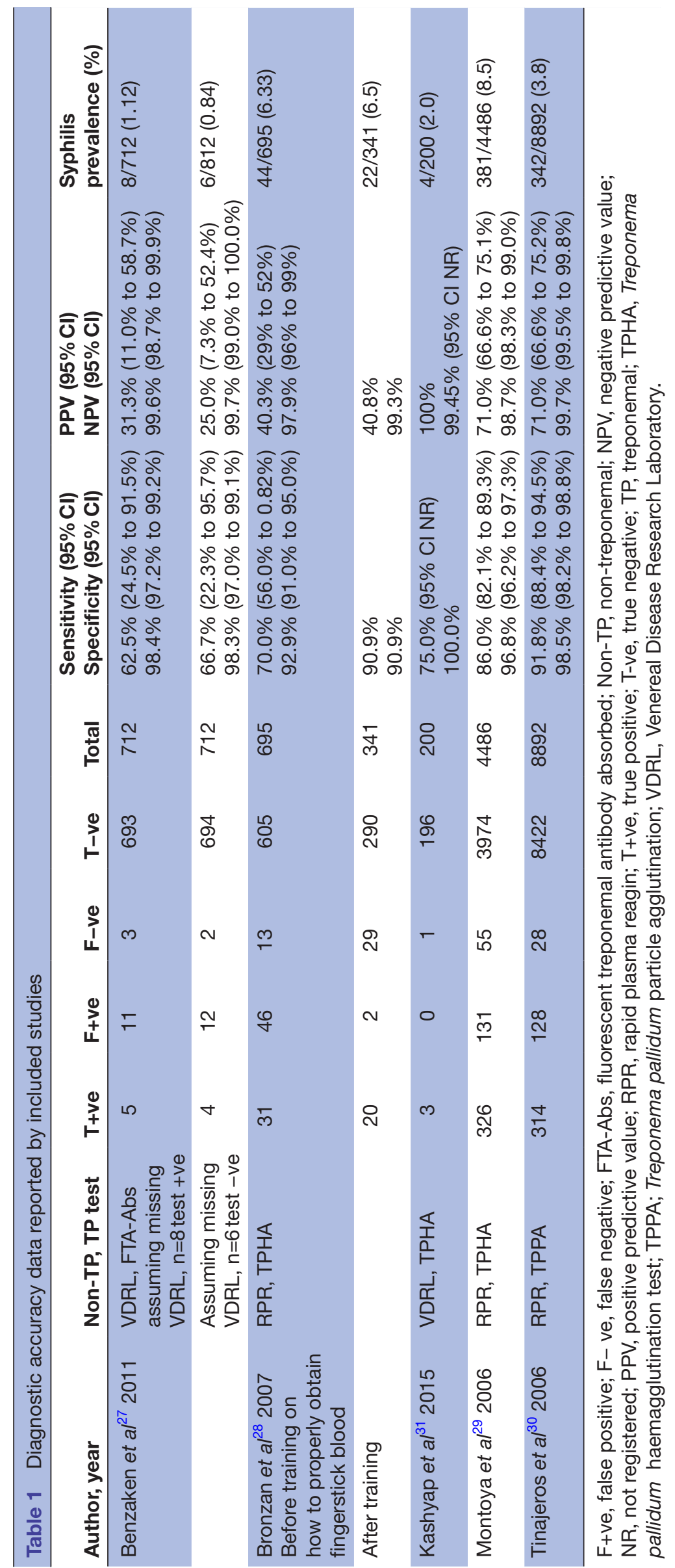




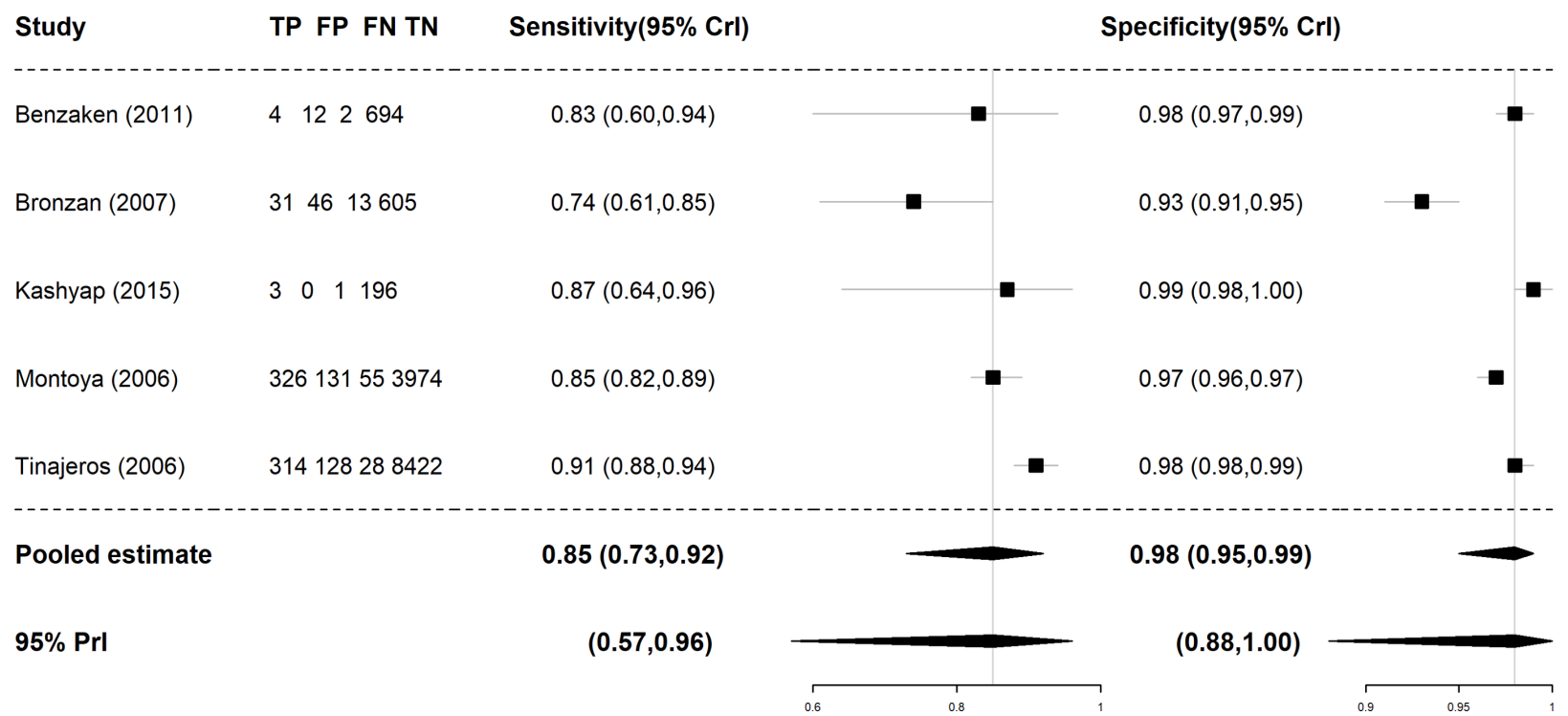

Figure 3 Sensitivity and specificity of ICS point-of-care compared with a standard reference test. FN, false negative; FP, false positive; ICS, immunochromatographic syphilis; Prl, prediction interval; TN, true negative; TP, true positive.

it was also not clear how VDRL-negative/TPHA-positive tests were interpreted, which could potentially influence the performance results.

The present review only included studies that used the conventional algorithm of dual reference standard
(non-TP and TP tests) as a reference standard. This is in contrast to the review by Tucker $e t a l^{17}$ that included studies of one TP test or an additional non-TP test and the review by Jafari et $a l^{18}$ that included studies of TP tests, non-TP-specific tests or TP and non-TP-specific tests (the

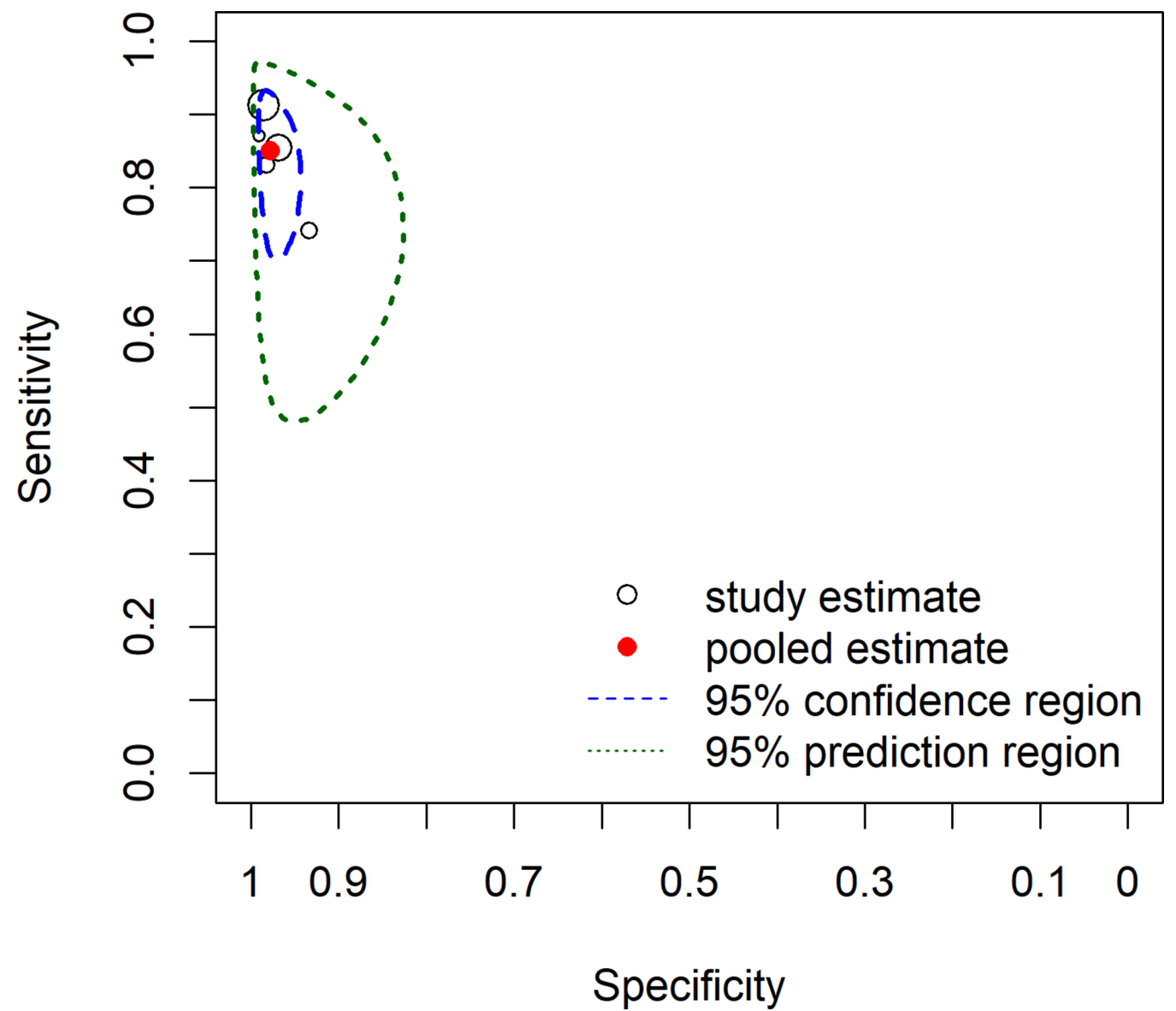

Figure 4 Joint sensitivity and specificity plot of immunochromatographic syphilis point-of-care compared with a standard reference test. 
inverse algorithm) but only presented a meta-analysis of studies evaluating a TP-specific reference standard test.

In comparison with the previous review by Tucker et $\mathrm{al}^{17}$ that included studies in women including sex workers attending STI clinics as well as antenatal clinics, the present review only included studies in pregnant women attending at any care settings (antenatal care settings, primary care settings, maternity hospital). In comparison with the previous review by Jafari $e t l^{18}$ that included studies in women attending antenatal clinic and sexually transmitted disease (STD) clinics. These differences in included studies are due to the main objective of the present review being to investigate the performance of on-site tests as part of prevention strategy of mother-to-child transmission of syphilis.

The review by Tucker et $a l^{17}$ reported that although there were variations in ICS test sensitivity across the included studies, that this variation was similar to sensitivity noted in traditional non-TP testing where sensitivity ranged from $78 \%$ to $100 \% .{ }^{17}$ From the meta-analysis in the review by Jafari $e t a l,{ }^{18}$ the authors observed that rapid and POC TP tests had varied sensitivity $(74.26 \%-$ $86.34 \%)$ ) and high specificity (95.85\%-99.43\%) (serum and blood) that were equal or better than laboratory-based non-TP tests. Jafari et $\mathrm{l}^{18}$ synthesised results from individual studies separately for each index test, using a Bayesian hierarchical summary receiver operating characteristic curve model and adjusted for imperfect reference standards.

Another difference between ours and these previous reviews is that for the Bronzan $e t a l^{28}$ study (included in the reviews by Tucker et $a l^{17}$ and Jafari et $\left.a l\right),{ }^{18}$ we used the on-site ICS results for all the study participants ( $\mathrm{n}=695$ ), which was considered to be more consistent with the results of the other included studies, rather than the on-site ICS results after training on how to properly obtain fingerstick blood $(\mathrm{n}=341)$.

Our searches identified two studies ${ }^{27} 31$ that were published after the searches of previous reviews in the area. $^{1718}$

The update search also identified the recent systematic review and meta-analysis by Rogozińska $e t a l,{ }^{34}$ which included two additional studies evaluating a qualitative RPR test as the index test. ${ }^{35} 36$

There were several differences in the QUADAS assessment reported by Rogozińska et $a l^{34}$ compared with the present systematic review. Notable differences were that Kashyap $e t a l^{11}$ was judged as high risk of bias and high concern for patient selection in the present review (unclear and low risk in Rogozińska et al), ${ }^{34}$ and that Montoya et $a l^{29}$ was judged at high risk of bias for study flow and timing in the present review (low risk in Rogozińska $e t a l) .{ }^{34}$ Two reviewers were involved in the QUADAS assessment in Rogozińska et al, ${ }^{34}$ whereas three reviewers (CNPRC, SH and MM-SJ) were involved in the present review.

The meta-analysis conducted by Rogozińska et $a e^{4}$ differed to that presented in the current review. Separate meta-analyses were conducted according to the specific ICS test implementation (Determine, VisiTect, SD Bioline). Since a frequentist estimation framework was used, this meant that there were too few studies to fit a bivariate model in the case of two of the ICS implementations (Determine, VisiTect) and so separate meta-analyses were conducted for sensitivity and specificity. For the current review, the synthesis was conducted under the assumption that different specific implementations of ICS rapid tests can be considered equivalent, and therefore combined in single meta-analysis model. The resulting pooled estimates represent the sensitivity/specificity of a general ICS test.

Public health strategies to prevent and treat maternal and fetal syphilis include early identification of infected individuals and high-risk populations, adequate treatment, identification of infected partners and their treatment, modification of high-risk behaviour and promoting the accessibility to and the use of healthcare. ${ }^{1}$ However, access to testing and implementing screening programmes varies significantly between countries and between rural and urban areas. 'In primary healthcare settings, there are often technical difficulties associated with performing serological tests, such as retaining trained personnel and assuring that supplies are adequate and tests are of good quality'. Additionally, if pregnant women fail to return for laboratory results or they are lost in transit, the treatment is delayed or missed.

\section{CONCLUSIONS}

This systematic review and meta-analysis observed that rapid ICS POC syphilis tests have a high sensitivity and specificity when performed in pregnant women at antenatal clinics. However, the methodological quality of the existing evidence and clinical heterogeneity of participants across studies should be taken into consideration.

Further diagnostic test accuracy of ICS POC tests at antenatal clinics compared with reference standard test that are reported in line with Standards for Reporting of Diagnostic Accuracy Studies ${ }^{37}$ would be of benefit, along with studies of duplex TP and non-TP rapid and POC tests. Studies evaluating the diagnostic test accuracy of STD coinfections such as HIV/Syphilis would also be of benefit. Considering the global efforts to eliminate congenital syphilis, the free access to simultaneous screening for these infections during pregnancy, through separate or dual tests, is crucial to tackle mother-to-child transmission of HIV and syphilis. Further studies that estimate the coverage of screening programmes would also be beneficial to help reduce the reservoir of infections in communities to a level at which disease transmission cannot be sustained.

Acknowledgements Special thanks for Suzy Paisley and Simon Dixon, both at School of Health and Related Research (ScHARR), University of Sheffield, for their helpful support during the scope of this review.

Contributors CNPRC, SH and MM-SJ developed the protocol. CNPRC, DSM and RC searched for the evidence and extracted the data. Data were checked by SH and MM-SJ. CNPRC, SH and MM-SJ undertook the quality assessment. JH undertook 
the statistical analysis. All authors were involved in preparing the final manuscript. CNPRC, DSM, SH, MM-SJ and JH addressed the peer-review comments.

Funding Funding was provided by the National Council for Scientific and Technological Development (CNPq), Ministry of Science, Technology, Innovation and Communication (no. 573642/2008-7; Ministry of Education/CAPES (no. 573642/2008-7); Research Foundation of the State of Rio de Janeiro (FAPERJ) (no. 573642/2008-7) and Oswaldo Cruz Foundation (Fiocruz). The funders had no role in study design, data collection and analysis, decision to publish, or preparation of the manuscript.

Competing interests None declared.

Patient consent Not applicable.

Provenance and peer review Not commissioned; externally peer-reviewed.

Data sharing statement № additional data are available.

Open Access This is an Open Access article distributed in accordance with the Creative Commons Attribution Non Commercial (CC BY-NC 4.0) license, which permits others to distribute, remix, adapt, build upon this work non-commercially, and license their derivative works on different terms, provided the original work is properly cited and the use is non-commercial. See: http://creativecommons.org/ licenses/by-nc/4.0/

(c) Article author(s) (or their employer(s) unless otherwise stated in the text of the article) 2018. All rights reserved. No commercial use is permitted unless otherwise expressly granted.

\section{REFERENCES}

1. Genç M, Ledger WJ. Syphilis in pregnancy. Sex Transm Infect 2000;76:73-9.

2. Avelleira JCR, Bottino G. Sífilis: diagnóstico, tratamento e controle. An Bras Dermatol 2006;81:111-26.

3. Kingston M, French $\mathrm{P}$, Goh $\mathrm{B}$, et al. UK National guidelines on the management of syphilis 2008. Int J STD AIDS 2008;19:729-40.

4. Klausner JD. The sound of silence: missing the opportunity to save lives at birth. Bull World Health Organ 2013;91:158-A.

5. World Health Organization. Global Health Observatory (GHO) data. Sexually Transmitted Infections (STIs). 2012 http://www.who.int/gho/ sti/en/ (accessed 6 Jun 2017).

6. Arnesen L, Serruya S, Duran P. Gestational syphilis and stillbirth in the Americas: a systematic review and meta-analysis. Rev Panam Salud Publica 2015;37:422-9.

7. Pan American Health Organization. 2010 Situation analysis: elimination of mother-to-child transmission of HIV and congenital syphilis in the Americas. Washington, D.C: Pan American Health Organization, 2011.

8. Peeling RW, $\mathrm{Ye} \mathrm{H}$. Diagnostic tools for preventing and managing maternal and congenital syphilis: an overview. Bull World Health Organ 2004;82:439-46.

9. Sato NS, de Melo CS, Zerbini LC, et al. Assessment of the rapid test based on an immunochromatography technique for detecting anti-Treponema pallidum antibodies. Rev Inst Med Trop Sao Paulo 2003;45:319-22.

10. Kamb M. Guidance on syphilis testing in Latin America and the Caribbean: improving uptake, interpretation and quality of testing in different clinical settings. Washington, DC: Pan American Health Organization, 2015.

11. Fears MB, Pope V. Syphilis fast latex agglutination test, a rapid confirmatory test. Clin Diagn Lab Immunol 2001;8:841-2.

12. Sato NS. Laboratorial Diagnosis of Syphilis. Syphilis - recognition, description and diagnosis. São Paulo, Brazil: Center of Immunology, Institute Adolfo Lutz, 2011.

13. Schackman BR, Neukermans CP, Fontain SN, et al. Costeffectiveness of rapid syphilis screening in prenatal HIV testing programs in Haiti. PLoS Med 2007;4:e183-47.
14. WHO. The use of rapid syphilis tests. Geneva, Switzerland: WHO/ TDR, 2006.

15. Kettler H, White K, Hawkes S. Mapping the landscape of diagnostics for sexually transmitted infections: Key findings and recommandations. Geneva, Switzerland: WHO/TDR, 2004.

16. Mabey D, Peeling RW. Syphilis, still a major cause of infant mortality. Lancet Infect Dis 2011;11:654-5.

17. Tucker JD, Bu J, Brown LB, et al. Accelerating worldwide syphilis screening through rapid testing: a systematic review. Lancet Infect Dis 2010;10:381-6.

18. Jafari Y, Peeling RW, Shivkumar S, et al. Are Treponema pallidum specific rapid and point-of-care tests for syphilis accurate enough for screening in resource limited settings? Evidence from a metaanalysis. PLoS One 2013;8:e54695.

19. Moher D, Liberati A, Tetzlaff J, et al. Preferred reporting items for systematic reviews and meta-analyses: the PRISMA statement. Ann Intern Med 2009;151:264-9.

20. Whiting PF, Rutjes AW, Westwood ME, et al. QUADAS-2: a revised tool for the quality assessment of diagnostic accuracy studies. Ann Intern Med 2011;155:529-36.

21. Reitsma JB, Glas AS, Rutjes AW, et al. Bivariate analysis of sensitivity and specificity produces informative summary measures in diagnostic reviews. J Clin Epidemiol 2005;58:982-90.

22. Macaskill P, Constantine Gatsonis JD, Roger Harbord YT. Chapter 10: Analysing and Presenting Results. Cochrane Handbook for Systematic Reviews of Diagnostic Test Accuracy Version 1.0. London: Cochrane Training, 2010:1-61.

23. R Core Team. R: A Language and Environment for Statistical Computing. Vienna, Austria: the R Foundation for Statistical Computing, 2016.

24. Lunn DJ, Thomas A, Best N, et al. WinBUGS - A Bayesian modelling framework: concepts, structure, and extensibility. Stat Comput 2000;10:325-37.

25. Sturtz S, Ligges U, Gelman A. R2WinBUGS : A Package for Running WinBUGS from R. J Stat Softw 2005;12.

26. Brooks SP, Gelman A. General methods for monitoring convergence of iterative simulations. J Comput Graph Stat 1998;7:434-55.

27. Benzaken AS, Sabidó M, Galban E, et al. Field performance of a rapid point-of-care diagnostic test for antenatal syphilis screening in the Amazon region, Brazil. Int J STD AIDS 2011;22:15-18.

28. Bronzan RN, Mwesigwa-Kayongo DC, Narkunas D, et al. On-site rapid antenatal syphilis screening with an immunochromatographic strip improves case detection and treatment in rural South African clinics. Sex Transm Dis 2007;34:S55-60.

29. Montoya PJ, Lukehart SA, Brentlinger PE, et al. Comparison of the diagnostic accuracy of a rapid immunochromatographic test and the rapid plasma reagin test for antenatal syphilis screening in Mozambique. Bull World Health Organ 2006;84:97-104.

30. Tinajeros F, Grossman D, Richmond K, et al. Diagnostic accuracy of a point-of-care syphilis test when used among pregnant women in Bolivia. Sex Transm Infect 2006;82(Suppl 5):v17-21.

31. Kashyap B, Sagar T, Kaur IR, et al. Utility of immunochromatographic assay as a rapid point of care test for screening of antenatal syphilis. Indian J Sex Transm Dis 2015;36:162-5.

32. World Bank. World Bank country and lending groups. Country classification. 2017 https://datahelpdesk.worldbank.org/ knowledgebase/articles/906519 (accessed 6 Jun 2017).

33. Peeling RW, Mabey D, Herring A, et al. Why do we need qualityassured diagnostic tests for sexually transmitted infections? Nat Rev Microbiol 2006;4:909-21.

34. Rogozińska E, Kara-Newton L, Zamora JR, et al. On-site test to detect syphilis in pregnancy: a systematic review of test accuracy studies. BJOG 2017;124:734-41.

35. Delport SD. On-site screening for maternal syphilis in an antenatal clinic. S Afr Med J 1993;83:723-4.

36. Van Dyck E, Van de Velden L, Ndoye I, et al. Evaluation of the rapid plasma reagin "teardrop" card test for screening of syphilis in field conditions. Sex Transm Dis 1993;20:194-7.

37. Bossuyt PM, Reitsma JB, Bruns DE, et al. STARD 2015: an updated list of essential items for reporting diagnostic accuracy studies. Clin Chem 2015;61:1446-52. 